\title{
A rare case of calcinosis cutis in rheumatoid arthritis
}

\begin{abstract}
Calcinosis cutis is a rare disorder characterized by of deposition of insoluble calcium salts in the skin and subcutaneous tissue. Five subtypes of calcinosis cutis are described: dystrophic, metastatic, idiopathic, iatrogenic, and calciphylaxis. ${ }^{1}$

Of these subtypes, dystrophic calcinosis (DC) is the most common, and it is the most frequently seen in association with underlying autoimmune connective tissue diseases. ${ }^{2}$ Dermatomyositis, systemic sclerosis and less commonly systemic lupus erythematous were described to be complicated by DC. However, DC associated with rheumatoid arthritis (RA) is extremely rare. ${ }^{2}$

The condition causes substantial morbidity and is associated with pain and limitation of movement when the process involves areas close to joints or when ulceration occurs. ${ }^{2} \mathrm{We}$ report a middle age Sudanese woman with good controlled RA who developed dystrophic calcinosis cutis.
\end{abstract}

Keywords: rheumatoid arthritis, calcinosis cutis
Volume 12 Issue 6 - 2020

\author{
Wafaa Hassan Ahmed Albashir, ${ }^{1,2}$ Rihab \\ Mohamed Hassan Ali,' Sufian Khalid M Nor, ${ }^{3}$ \\ Sara yassin, ${ }^{4,5}$ Mosab Nouraldein Mohammed \\ Hamad $^{6}$ \\ 'Friendship teaching hospital, Sudan \\ ${ }^{2}$ Department of rheumatology, Aliaa Speciality hospital, Sudan \\ ${ }^{3}$ Department of Medicine, Faculty of Medicine, Nile Valley \\ University, Sudan \\ ${ }^{4}$ Imperial hospital, Khartoum, Sudan \\ ${ }^{5}$ Alsaaha specialized hospital, Khartoum, Sudan \\ 6Department of Medical Parasitology, Faculty of Health Science, \\ Elsheikh Abdallah Elbadri University, Sudan
}

Correspondence: Mosab Nouraldein Mohammed Hamad, Department of Medical Parasitology, Faculty of Health Science, Elsheikh Abdallah Elbadri University, Sudan,

Email musabnoor3@gmail.com

Received: November 06, 2020 | Published: December 02, 2020

\section{Case details}

A 50 years Sudanese RA patient was referred from the GP as she experienced scattered skin hardening on the right thigh which extended to the buttock which recently caused mild limitation of movement of her right hip joint. She felt that the hardening is beneath her skin with progressive darkening of the overlying skin during the last year. The hardening and darkening progressed to involve the left hip, right thigh, right knee and the upper right chest. The described areas were non pruritic and the patient described discomfort rather than pain.

Her GP thought about morphed and referred the patient to the rheumatologist. The patient was diagnosed as rheumatoid arthritis 5 years prior to the current presentation based on symptoms of significant early morning stiffness and chronic symmetrical polyarthritis affecting mainly small joints of her hands and feet. She had also fatigue and low grade fever. Her blood tests at that time showed elevated acute phase reactants and high levels of Anti citrullinated protein antibodies $30 \mathrm{u}$ $\mathrm{ml}$ (normal less than $20 \mathrm{u} / \mathrm{ml}$ ).

Her diagnosis of rheumatoid arthritis was made by a rheumatologist based on EULAR/ACR 2010 criteria. She had regular follow up and remained on remission in most of her follow up visits and of low disease activity in some visits according to DAS28.

Her rheumatoid arthritis was controlled on methotrexate $(15 \mathrm{mg}$ / week), hydroxyl chloroquine (200mg/day) and folic acid $(10 \mathrm{mg} /$ week) as a treatment regime. She was off glucocorticoids and takes NSAIDS per need. She patient was complaint to the medications.

The patient did not describe new small joint complaint, nor muscle pain or weakness. There is no Raynaud phenomenon, dry eyes or mouth, hair fall, skin rash, mouth or genital ulcers and did not have significant constitutional symptoms or neurologic deficits.

No recent complaints suggestive of GI, Cardiopulmonary, neurological insolvents or genitourinary involvement.

She did not have any concurrent diseases such as diabetes or hypertension, and did not smoke or consume alcohol. She has no family history of connective tissue disease.

She led an active lifestyle with regular meals containing daily requirements of calcium and minerals and refused to take extra calcium supplementations because if constipation caused by calcium containing pills.

\section{On examining the patient}

Normal vital signs, joints show no deformities or synovitis. There are no signs of sclerodactyly, telangiectasia. Skin hyperpigmentation involving the lateral and anterior aspects or the right thigh up to the right buttock and extending to the right knee and the lateral aspect of the left thigh.

There are hyper pigmented indurated plaques with atrophic surfaces ranging from $1 \mathrm{~cm}$ to $30 \mathrm{~cm}$ are firmly attached to the underlying structure (hardening extends to the depth is of average of $1-2.5 \mathrm{~cm}$ ). Cardiopulmonary and GI systems were normal on examination and neurological examination (including muscle power was normal)

Current DAS28 (CRP): 1.51 (remission).

\section{Laboratory studies}

Were as follows:

$\mathrm{Hb} 11.3 \mathrm{mg} / \mathrm{dl}$ with normal differential 
Parathyroid hormone: 45.6pg./ml (normal 10-65pg./ml)

Serum calcium: $8.5 \mathrm{mg} / \mathrm{dl}$

Total protein: $6 \mathrm{~g} / \mathrm{dl}$

Albumin: $4.5 \mathrm{~g} / \mathrm{dl}$

Serum phosphorus: $3.3 \mathrm{mg} / \mathrm{dl}$

24-hour urine calcium: $55 \mathrm{mg} /$ day

Serum creatinine: $1 \mathrm{mg} / \mathrm{dl}(0.6-1.4 \mathrm{mg} / \mathrm{dl})$

Blood urea: 12-20

Alkaline phosphatase: $76 \mathrm{IU} / \mathrm{L}$

Erythrocyte sedimentation rate: $50 \mathrm{~mm} / \mathrm{h}$

C-reactive protein: $2.1 \mathrm{mg} / \mathrm{dl}$

Antinuclear antibody: negative.

Anti-citrullinated protein antibodies: $30 \mathrm{u} / \mathrm{ml}$ (normal less than $20 \mathrm{u} / \mathrm{ml})$.

\section{Imaging}

AP radiograph of the pelvis showed extensive subcutaneous calcifications involving the gluteal regions and the lateral aspect of the upper both thighs. The visualized muscle bundles appear free. Bones are unremarkable. AP and lateral radiograph of the RT knee shows florid flakes of calcifications within the subcutaneous fat planes with no evidence of muscle or tendon calcification. All features are in keeping with calcinosis cutis.

\section{Discussion}

The likelihood of developing DC varies among the autoimmune connective tissue diseases, as no specific incidence and frequency are available and no sex predilection is documented. However, it's commonly occurs in patients with systemic sclerosis, especially the limited form (CREST) and dermatomyocitis. It is reported that 25 to $40 \%$ of patients with limited systemic sclerosis will develop calcinosis cutis ten years after the onset of disease, ${ }^{3}$ while $30 \%$ of adults and up to $70 \%$ of children and adolescents with dermatomyocitis will experience DC. $33 \%$ of systemic lupus erythematosus patients can present with periarticular calcification and $17 \%$ with soft tissue calcification. ${ }^{4}$ However, there are only few cases of calcinosis cutis related to RA in the literature.

Our patient has a relatively short duration, well-controlled rheumatoid arthritis that experienced an extensive DC with only mild discomfort symptoms and minimal limitation of right hip joint movement with no complications like ulceration or septic arthritis as reported in few case reports.

After discussion with a multidisciplinary team, we decided to treat the patient with Diltiazem as it decreases the amount of calcium that enters cells and macrophages of the damaged tissues. High doses at $2 \mathrm{mg} / \mathrm{kg} / \mathrm{d}$ to $4 \mathrm{mg} / \mathrm{kg} / \mathrm{d}$ are necessary for therapeutic response. We also added small dose of Colchicine due to its anti-inflammatory properties. The patient showed good response to treatment in the subsequent visits.

Different treatment options have been tried in the literature with no proven efficacy. Depending on the underlying cause, a multidisciplinary team of physicians including nephrologist, rheumatologist, and hematologist may be needed to manage the condition. Medical therapy may be used to help relieve symptoms of the condition but are generally of limited and variable benefit. ${ }^{5}$

Medications that may be tried include corticosteroids, probenecid, colchicine, sodium etidronate, diphosphonates, diltiazem, and magnesium and aluminium. Smaller lesions have been reported to respond to warfarin, ceftriaxone, and intravenous immunoglobulin (IVIG). ${ }^{5}$

Larger lesions respond to diltiazem, bisphosphonates, probenecid, aluminum hydroxide and surgical excision or curettage. ${ }^{5}$ Patients with small and localized lesions are good candidates for surgical treatment whereas more generalized disease will require medical management. ${ }^{5}$

As our patient has experienced DC we started on high dose of Diltiazem based on its effect in decreasing the amount of calcium that enters cells and macrophages of the damaged tissues. A small dose of colchicine was added due to its anti-inflammatory properties. Our patient showed considerable improvement in her subsequent visits.

\section{Diagnosis and treatment}

MDT appreciated was done and we interpreted the patient's condition as calcinosis cutis associated with RA. A dose of $2 \mathrm{mg} / \mathrm{kg}$ of Diltiazem and a small dose of colchicine were started for long term treatment. Surgical treatment for the lesions around the right hip which recently cause limitation of movement was also discussed with the patient, but fortunately it was not needed owing to good response to medical treatment (Figure 1-3).

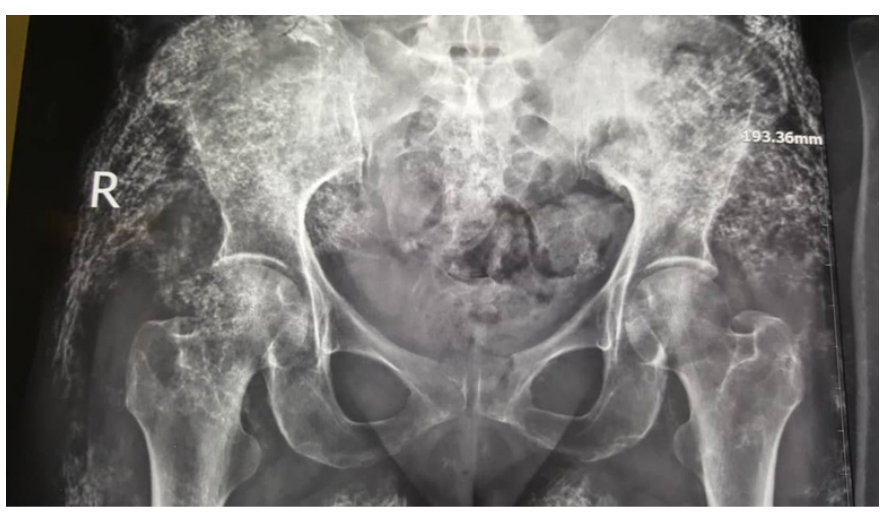

Figure I AP radiograph of the pelvis showed extensive subcutaneous calcifications.

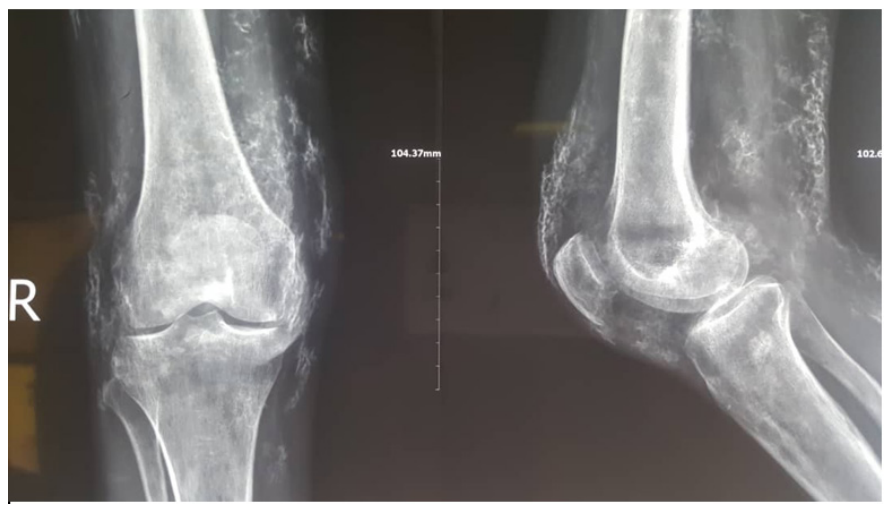

Figure $\mathbf{2}$ Lateral radiograph of the RT knee shows florid flakes of calcifications. 


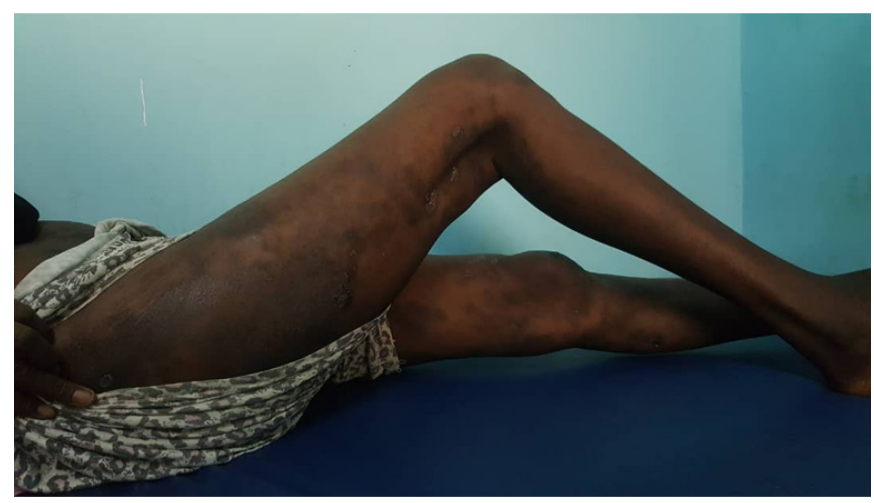

Figure 3 Wide areas of skin hyperpigmentation with hyper pigmented indurated plagues and atrophic surfaces.

\section{Conclusion}

We conclude that medications used such as include corticosteroids, probenecid, colchicine, sodium etidronate, diphosphonates, diltiazem, and magnesium and aluminium, where satisfy to treat this case, and no need for surgical intervention. Patient will follow up to make ensure from his cure.

\section{Acknowledgments}

None.

\section{Conflicts of interest}

The authors declare there are no conflicts of interest.

\section{Funding}

None.

\section{References}

1. Reiter N, El-Shabrawi L, Leinweber B, et al. Calcinosis cutis: part I. Diagnostic pathway. J Am Acad Dermatol. 2011;65(1):1-12.

2. Balin SJ, Wetter DA, Andersen LK, et al. Calcinosis cutis occurring in association with autoimmune connective tissue disease: the Mayo Clinic experience with 78 patients, 1996-2009. Arch Dermatol. 2012;148(4):455-462.

3. Mark F Hoeltzel, Edward J Oberle, Angela Byun Robinson, et al. The presentation, assessment, pathogenesis, and treatment of calcinosis in juvenile dermatomyositis. Curr Rheumatol Rep. 2014;16(12):467.

4. Le C, Bedocs PM. Calcinosis Cutis. Treasure Island: StatPearls Publishing; 2020.

5. https://healthjade.net/crest-syndrome/ 